\title{
Randomized Comparison of Cavotricuspid Isthmus Ablation for Atrial Flutter Using an Open Irrigation-Tip versus a Large-Tip Radiofrequency Ablation Catheter
}

\author{
KARL J. ILG, M.D., ${ }^{*}$ MICHAEL KÜHNE, M.D., ${ }^{*}$ THOMAS CRAWFORD, M.D., \\ AMAN CHUGH, M.D., KRIT JONGNARANGSIN, M.D., ERIC GOOD, D.O. FRANK PELOSI, JR, \\ M.D., FRANK BOGUN, M.D., FRED MORADY, M.D., and HAKAN ORAL, M.D.
}

From the Division of Cardiovascular Medicine, University of Michigan, Ann Arbor, Michigan, USA

\begin{abstract}
Ablation of Typical Atrial Flutte. Background: Large-tip (10 mm) catheters (LTCs) and openirrigation-tip catheters (OITCs), both capable of creating large lesions, are more effective than conventional catheters for cavotricuspid isthmus (CTI) ablation. However, it is not clear whether complete CTI block can be achieved more efficiently using an LTC or an OITC. The purpose of this study was to compare the efficiency of radiofrequency catheter ablation (RFA) of the CTI using LTC versus OITC to eliminate atrial flutter (AFL).

Methods and Results: Sixty consecutive patients (age $=62 \pm 10$ years) with typical AFL were randomized to undergo RFA of CTI using an LTC $(10 \mathrm{~mm})$ or an OITC. If complete CTI block was not achieved by $\leq 30$ minutes of RFA, patients were allowed to cross over to ablation with the other catheter. A 3-dimensional electroanatomical mapping system was used for catheter navigation only with the OITC. The mean duration of RFA to achieve CTI block in $50 \%$ of the patients was $6.8 \pm 2.2$ minutes with an LTC and $11.7 \pm 2.7$ minutes with an OITC $(P=0.001)$. After 30 minutes of RFA, CTI block was achieved in $26 / 30(87 \%)$ and $25 / 30$ patients $(83 \%)$ using an LTC and an OITC, respectively $(P=1.0)$. After crossover, CTI block was achieved in $4 / 5(80 \%)$ and in $4 / 4$ patients $(100 \%)$ with an LTC and OITC, respectively $(P=1.0)$. LTC was associated with a lower volume of intravenous fluid administration $(388 \pm 365 \mathrm{~mL}$ versus $865 \pm 451 \mathrm{~mL}, P=0.0001)$ and a trend for shorter procedure duration $(95 \pm 31$ minutes versus $114 \pm 50$ minutes, $P=0.09)$ than the OITC. At $6 \pm 3$ months, 30/30 patients $(100 \%)$ in the LTC and $27 / 30$ patients $(90 \%)$ in the OITC groups remained free from $A F L$, respectively $(P=0.24)$. Except for one inconsequential steam-pop during RFA with the OITC, there were no complications.

Conclusions: Complete CTI block is achieved more rapidly using an LTC than an OITC, and with a similar clinical efficacy. (J Cardiovasc Electrophysiol, Vol. 22, pp. 1007-1012, September 2011)

atrial flutter, catheter ablation, cavotricuspid isthmus, irrigated-tip catheter, monitoring
\end{abstract}

Radiofrequency catheter ablation (RFA) is highly effective in eliminating cavotricuspid isthmus (CTI)-dependent atrial flutter (AFL), and often is the treatment of choice for patients with AFL.$^{1-4}$ Large-tip and irrigated-tip catheters, both capable of creating larger lesions than conventional catheters, have been widely used to negate the technical challenges in creating transmural lesions along the complex structure of

${ }^{*} \mathrm{KI}$ and MK contributed equally to this paper.

Supported in part by a grant from Boston Scientific.

Michael Kühne was supported by the Swiss National Science Foundation; he reports educational grants from Biosense Webster and Boston Scientific. Hakan Oral reports research support from St. Jude Medical; he serves as consultant to or on the advisory board of Medtronic-Ablation Frontiers. Other authors: No disclosures.

Address for correspondence: Hakan Oral, M.D., Cardiovascular Medicine, SPC 5853, University of Michigan Health System, 1500 E. Medical Center Dr., Ann Arbor, MI 48109-5853, USA. Fax: 734-936-7026; E-mail: oralh@umich.edu

Manuscript received 24 November 2010; Revised manuscript received 31 January 2011; Accepted for publication 31 January 2011.

doi: $10.1111 / \mathrm{j} .1540-8167.2011 .02045 . \mathrm{x}$ the CTI. ${ }^{5-7}$ Prior studies have reported comparable acute and chronic efficacy rates with these catheters. 5,6,8-11 However, there are no data on the efficiency and efficacy of catheter ablation, that is, the ease with which complete conduction block is achieved when a $10-\mathrm{mm}$, large-tip catheter (LTC) versus an open-irrigation-tip catheter (OITC) is used.

The purpose of this prospective, randomized study was to compare the efficiency of CTI ablation to eliminate AFL using an LTC (10 mm) and OITC, and also to compare the clinical efficacy and safety of CTI ablation with these catheters.

\section{METHODS}

\section{Study Subjects}

The subjects of this study were 60 consecutive patients with CTI-dependent AFL who underwent RFA. The mean age was $62 \pm 10$ years (Table 1). There were 47 men and 13 women. The mean left ventricular ejection fraction was $0.53 \pm 0.13$. Patients who had a prior ablation procedure to eliminate atrial fibrillation or flutter were excluded from this study. Eight and 9 patients who underwent ablation with a LTC and OITC were noted to have infrequent episodes of paroxysmal atrial fibrillation prior to CTI ablation. 
TABLE 1

Clinical Characteristics

\begin{tabular}{lccc}
\hline & LTC N = 30 & OITC N = 30 & P \\
\hline Age (years) & $64 \pm 11$ & $60 \pm 8$ & 0.22 \\
Gender (M/F) & $25(83 \%)$ & $22(76 \%)$ & 0.53 \\
Left ventricular ejection fraction & $51 \pm 12$ & $55 \pm 14$ & 0.26 \\
Left atrial enlargement $(>40 \mathrm{~mm})$ & $19(63 \%)$ & $17(57 \%)$ & 0.79 \\
Hypertension & $20(67 \%)$ & $21(70 \%)$ & 1.00 \\
Structural disease & $6(20 \%)$ & $3(10 \%)$ & 0.47 \\
Congestive heart failure & $6(20 \%)$ & $8(27 \%)$ & 0.76 \\
\hline
\end{tabular}

Data are shown as mean \pm SD. Percent values are shown in parentheses.

\section{Study Protocol}

The study protocol was approved by the Institutional Review Board. Patients between 18 and 85 years of age with a history of typical AFL documented on a 12-lead ECG were enrolled in the study. Patients then were randomized 1:1 to undergo CTI ablation using either a 10-mm-tip RFA catheter (Blazer II XP, Boston Scientific, Boston, MA, USA) or a 3.5-mm-open-irrigation-tip RFA catheter (Thermocool ${ }^{\circledR}$, Biosense Webster, Diamond Bar, CA, USA) using a closedenvelope randomization schedule before the procedure. The clinical characteristics of the 2 groups were similar (Table 1).

\section{Electrophysiologic Study and RFA}

All patients provided written informed consent. Antiarrhythmic drugs were discontinued $>5$ half-lives prior to the procedure, with the exception of amiodarone, which was discontinued 2 months before the procedure. Vascular access was obtained through a femoral vein. After an initial dose of 3,000 units of heparin, additional 1,000 units of heparin were administered for every additional hour of the procedure.

A decapolar catheter (Biosense Webster) was positioned in the coronary sinus. Based on the randomization scheme, a LTC or an OITC was positioned along the CTI under fluoroscopic guidance. A long sheath (SR0, St. Jude Medical, Minnetonka, MN, USA) was used in all patients to facilitate catheter stability and contact. Intracardiac electrograms were filtered at band-pass settings of $30-500 \mathrm{~Hz}$ and were stored digitally (EP Med Systems Inc., West Berlin, NJ, USA). Pacing was performed at twice the diastolic threshold using an electronic stimulator (EP Med Systems Inc.).

RFA was performed during AFL until the tachycardia terminated and then during pacing from the coronary sinus until complete conduction block was achieved in 22 of 60 patients who presented in AFL. RFA was performed during pacing from the coronary sinus in the remaining 38 patients who presented in sinus rhythm. Radiofrequency energy was applied for 30 seconds or until loss of local electrograms at each site along the CTI during a continuous pullback from the ventricular aspect to the inferior vena cava.

\section{CTI ablation using an LTC}

In patients randomized to ablation using an LTC, RFA was performed under fluoroscopic guidance. The LTC was positioned at the ventricular aspect of the CTA at the 6 o'clock position in the $\mathrm{LAO} 45^{\circ}$ projection. The LTC was gradually withdrawn to the caval aspect of the CTI during applications of radiofrequency energy. If complete block was not achieved, the catheter was repositioned at the ventricular side of the CTI and the ablation line was mapped to identify gaps. If complete block still was not achieved after the second pass, then a new line on the medial or lateral aspect of the first line was created. Radiofrequency energy was delivered at a maximum of 100 Watts and a maximum temperature of $60^{\circ} \mathrm{C}$ (Maestro, Boston Scientific, Natick, MA).

\section{CTI ablation using an OITC}

In patients randomized to ablation using an OITC, the ablation catheter was positioned along the CTI similar to when an LTC was used as described above. Radiofrequency energy was delivered at a maximum temperature of $48^{\circ} \mathrm{C}$ and a maximum power of 40 Watts at a flow rate of $30 \mathrm{~mL} / \mathrm{min}$ (Stockert, Biosense Webster). In patients randomized to ablation using an OITC catheter, a 3-dimensional electroanatomical mapping system (CARTO, Biosense-Webster) was routinely used for catheter navigation.

The endpoint of the ablation procedure was complete bidirectional conduction block across the CTI. Clockwise conduction block was confirmed by the presence of parallel and widely split (>110 ms) double potentials along the CTI and also by reversal of the activation sequence as assessed by high-density mapping lateral to the ablation line during pacing from the coronary sinus. ${ }^{4}$ Counterclockwise conduction block was confirmed by assessing the same parameters during pacing from the low right atrium.

If complete conduction block was not achieved with up to 30 minutes of RF energy delivery, patients were allowed to cross over to ablation with the other catheter.

\section{Study Endpoints}

The primary endpoint of the study was the efficiency of RF ablation to achieve complete conduction block across the CTI. To accurately analyze the time course, mean duration of RFA to achieve complete block in 50\% of patients using an LTC or an OITC was compared. The cumulative proportion of patients in whom complete block was achieved at 2.5-minute intervals of RF energy delivery also was determined.

The secondary endpoints of the study were: (1) acute efficacy within 30 minutes of RF energy application; (2) fluoroscopy time to complete CTI conduction block; (3) total procedure time; (4) clinical efficacy, defined as freedom from AFL in the absence of antiarrhythmic drug therapy; and (5) peri- and post procedural complications.

\section{Follow-up}

Patients were discharged home the same day and were maintained on warfarin for 6 weeks unless there was another indication to continue warfarin. Patients were seen in an outpatient clinic 3 to 6 months after the ablation. They were instructed to call a dedicated nurse whenever they experienced symptoms suggestive of their arrhythmia. A 12-lead EKG was obtained during follow-up visits, and an auto-triggered event monitor was provided for 3 weeks to look for asymptomatic arrhythmias 3 months after the ablation.

\section{Statistical Analysis}

Continuous variables are expressed as mean \pm 1 standard deviation. Comparisons of continuous variables were performed using Student's $t$-test. Comparisons of categorical 


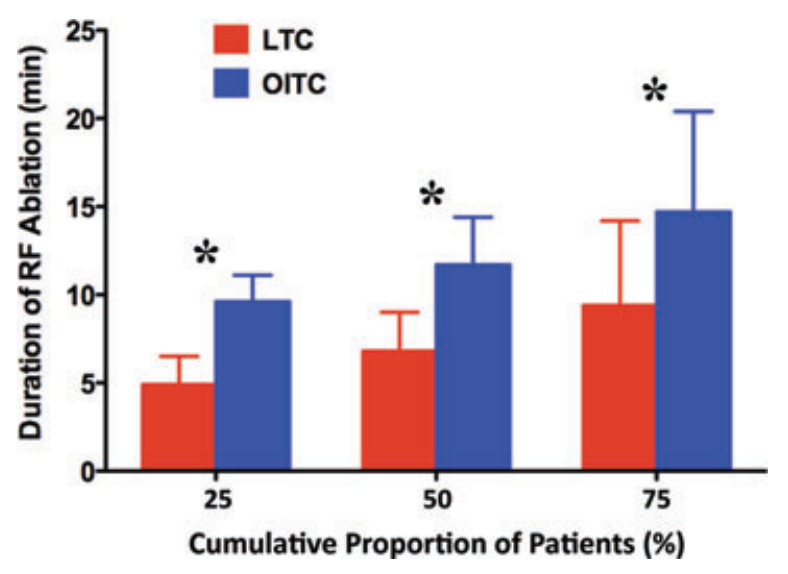

Figure 1. Mean duration of RF ablation to achieve complete block using an LTC (red) or OITC (blue) in $25 \%, 50 \%$, and $75 \%$ of the patients with AFL. ${ }^{*} P<0.05$.

variables were performed using Fisher's exact test. A P $<0.05$ indicated a statistical significance.

\section{RESULTS}

\section{Efficiency of CTI Ablation}

The mean duration of RFA to achieve complete CTI block in $50 \%$ of patients in each group was $6.8 \pm 2.2$ minute with an LTC and $11.7 \pm 2.7$ minute with an OITC $(\mathrm{P}=0.0001)$. CTI block was achieved in $75 \%$ of the patients in each group after $9.4 \pm 4.8$ minute of RFA using an LTC and after $14.7 \pm 5.7$ minute of RFA using an OITC $(\mathrm{P}=0.001$, Fig. 1).

CTI block was achieved with $\leq 10$ minutes of RFA in 14 of $30(47 \%)$ with an LTC and in 4 of $30,(13 \%)$ with an OITC ( $\mathrm{P}=0.01$, Fig. 2$)$; by $\leq 12.5$ minutes of RFA in 18 of $30(60 \%)$ with an LTC and in 9 of $30(30 \%)$ with an OITC $(\mathrm{P}=0.037)$; and by 15 minutes of RFA in 20 of $30(67 \%)$ with an LTC and in 13 of $30(43 \%)$ with an OITC $(\mathrm{P}=0.12)$. After 30 minutes of RFA, complete block was achieved in 26 of 30 patients $(87 \%)$ in the LTC and in 25 of 30 patients $(83 \%)$ in the OITC groups $(\mathrm{P}=1.0)$.

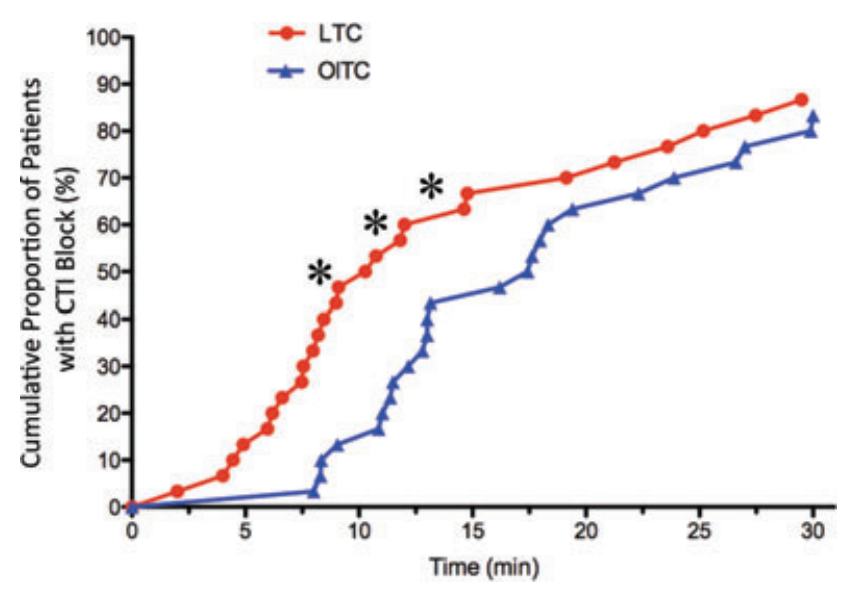

Figure 2. Duration of RF ablation required to achieve complete CTI block. Ablation with an LTC (red) resulted in complete CTI block in a larger proportion of patients sooner than with an OITC (blue). ${ }^{*} P<0.05$.

\section{Crossover}

After 30 minutes of RFA, complete conduction block was not achieved in 4 of 30 patients (13\%) with an LTC and in 5 of 30 patients $(17 \%)$ with an OITC $(\mathrm{P}=1.0)$. Per study protocol, these patients crossed over to ablation with the other catheter. After crossover, complete conduction block was achieved by $8.8 \pm 2.4$ minute of RFA using an LTC and by $7.9 \pm 1.7$ minute of RFA when using an OITC $(\mathrm{P}=0.61)$.

On an intention-to-treat analysis, after the crossovers, complete CTI block was achieved in 30 of 30 patients (100\%) who were randomized to RFA using an LTC and in 29 of 30 patients (97\%) who were randomized to RFA using an OITC $(\mathrm{P}=1.0)$.

\section{Procedure and Fluoroscopy Duration, and Amount of Intravenous Fluids Administered}

On an intention-to-treat analysis, the total procedure duration was $95 \pm 31$ minute in the LTC group and $114 \pm 50$ minute in the OITC $(\mathrm{P}=0.09)$. The total fluoroscopy time was $23.5 \pm 10.7$ minute in the LTC group, and $23.9 \pm 15.2$ minute in the OITC group $(\mathrm{P}=0.9)$. The total amount of intravenous fluids administered was $388 \pm 365 \mathrm{~mL}$ in the LTC and $865 \pm 451 \mathrm{~mL}$ in the OITC groups, respectively $(\mathrm{P}<0.001)$.

Among patients in whom CTI block was achieved with $\leq 30$ minutes of RFA, the total procedure duration was $89 \pm 22$ minute in the LTC group and $108 \pm 51$ minute in the OITC group $(\mathrm{P}=0.09)$. The total fluoroscopy time was $22.3 \pm 10.0$ minute in the LTC group, and $22.8 \pm 16.4$ minute in the OITC group $(\mathrm{P}=0.88)$. The total amount of intravenous fluids administered was $363 \pm 356 \mathrm{~mL}$ with an LTC and $796 \pm 436 \mathrm{~mL}$ with an OITC $(\mathrm{P}<0.001)$.

\section{Clinical Efficacy and Safety}

On an intention-to-treat analysis, at a mean follow-up of $6 \pm 3$ months, 30 of 30 patients (100\%) randomized to LTC and 27 of 30 patients (90\%) randomized to OITC were free from recurrent atrial flutter in the absence of antiarrhythmic drug therapy $(\mathrm{P}=0.24)$. There was no relationship between the duration of RFA required to achieve complete block and freedom from recurrent AFL during follow-up. Similarly, patients who crossed over to ablation with the alternate catheter had a similar clinical efficacy as the patients who did not have to cross over. There was a single tissue steam pop during RFA with the OITC. However there were no adverse consequences of this tissue pop. There were no other complications in this study.

\section{DISCUSSION}

\section{Main Findings}

The main findings of this study are: (1) Complete conduction block across the CTI is achieved more quickly with a LTC than an OITC; (2) By 30 minutes of RFA, complete CTI block is achieved in $\sim 85 \%$ of patients with either ablation catheter; (3) After crossover to ablation with the other catheter, complete conduction block is achieved in almost all patients with $<10$ minutes of additional RFA; (4) Clinical efficacy of CTI ablation to eliminate AFL using an LTC or OITC is similar and is $\geq 90 \%$; (5) RFA with an OITC is 
associated with $\sim 500 \mathrm{~mL}$ of additional intravenous fluid administration compared to LTC; (6) Use of a 3D electroanatomical mapping system with an OITC is not associated with an improvement in procedural efficiency or clinical efficacy or a decrease in fluoroscopy duration.

These findings indicate that an LTC is more efficient than OITC for achieving complete CTI block.

\section{LTC versus OITC}

With both LTC and OITC, cooling enables higher power delivery and larger lesions. Although longer segments can be ablated using an LTC, the ablation of deep recesses, where lack of sufficient blood flow may impede efficient tip cooling and power delivery, can be more difficult. ${ }^{7}$ On the other hand, with an OITC the length of ablation lesions may be shorter than with an LTC at other sites. Variations in the topography of the CTI are common and recesses can be observed in up to $13 \% .{ }^{12}$ It may be that complete CTI block is achieved more efficiently with an LTC than with an OITC because the majority of patients with AFL have a smooth CTI without deep recesses. ${ }^{12,13}$ However, in patients with CTI recesses, LTC may not be sufficient to create transmural lesions, explaining why CTI block was not achieved in all patients with 30 minutes of RFA with an LTC.

A prior study that assessed the CTI anatomy prior to ablation in all patients demonstrated that LTC (8-mm-tip) was associated with a shorter fluoroscopy and RF duration than OITC in only patients with a straight CTI. ${ }^{10}$ However, there was no statistically significant difference between the 2 catheters in patients with a concave or pouch-like CTI. Clinical follow-up on freedom from recurrent AFL was not reported in this study. In a subsequent study, ${ }^{11}$ patients were randomized to CTI ablation with or without CTI angiography. In the angiography group an LTC (8-mm-tip) or an OITC was selected based on CTI anatomy, and in the control group who did not undergo angiography an OITC was used in all patients. Consistent with the findings of the prior study, catheter selection based on CTI angiography was associated with a shorter fluoroscopy and RF application duration than empirical ablation using an OITC without angiography. Information on clinical follow-up was not provided in this study.

In the present study, a simple and practical approach was taken without having to perform CTI angiography, which could potentially increase the procedure duration, cost, radiation exposure, and add the risk of contrast nephropathy. In this study, regardless of the CTI anatomy, LTC was found to be more efficient than OITC in a consecutive series of patients with AFL who present to the electrophysiology laboratory.

\section{3-Dimensional Navigation and Catheter Irrigation}

In this study, navigation of an OITC using a 3D electroanatomical mapping system was not associated with an improvement in procedural efficiency or fluoroscopy exposure compared to navigation of an LTC only by conventional fluoroscopy. Ablation along the CTI involves linear lesions between well-defined anatomical landmarks with characteristic electrograms; therefore, $3 \mathrm{D}$ catheter navigation may not be facilitatory during CTI ablation.

In a prior randomized study, ${ }^{14} \mathrm{CTI}$ ablation using an OITC with or without a 3D electroanatomical mapping system was associated with similar acute and long-term efficacy and pro- cedure duration. Fluoroscopy exposure was shorter when a $3 \mathrm{D}$ navigation system was used, but at the expense of an increase in procedural cost.

In another randomized study, CTI ablation using an 8-mm tip catheter with high density electroanatomical mapping to avoid large voltage areas also had a similar acute and clinical efficacy to CTI ablation with conventional 3D navigation. ${ }^{15}$ However, because 3D navigation system was not used with an LTC and because ablation using an OITC was not performed without a 3D navigation system, a potentially helpful role of $3 \mathrm{D}$ catheter navigation, particularly with an LTC, cannot be ruled out based on the findings of this study.

As expected, ablation using an OITC was associated with additional $500 \mathrm{~mL}$ of saline infusion during the procedure. For the majority of patients, this much fluid should not be a concern. However, in patients with advanced heart failure, hypoalbuminemic states, or end-stage renal disease, the amount of fluid infusion should be considered in catheter selection.

\section{Efficacy and Safety}

Clinical efficacy of catheter ablation using an LTC or OITC were similar in this study, suggesting that once complete conduction block is achieved lesions are likely to be transmural and recovery of conduction is unlikely regardless of the ablation catheter used. The primary difference appears to be the duration of RFA to achieve complete block, but not clinical efficacy.

One of the major concerns with the use of LTC or OITC is steam-pops and myocardial perforation. ${ }^{16}$ Although attention to RF parameters and impedance monitoring are very helpful, at times there may be no warning signs and steam-pops can occur suddenly. In this study, there was one steam pop with the use of an OITC. However, there were no adverse events associated with the use of either catheter. Therefore, at the RF settings used in this study and with close monitoring of impedance and temperature, both catheters appear to be safe for ablation of CTI.

\section{Prior Studies}

In a prior study, an OITC was compared to a conventional $4 \mathrm{~mm}$ tip catheter and was found to be more effective than the conventional catheter. ${ }^{8}$ In another study, CTI ablation using an $8 \mathrm{~mm}$ tip catheter was found to be more effective than a $4 \mathrm{~mm}$ tip catheter. ${ }^{5}$ CTI ablation was performed using an OITC versus an 8-mm-gold-tip versus platinum-iridium-tip catheter in a prior randomized study. ${ }^{17}$ There were 20 patients in each group and all 3 catheters were found to be equally effective in achieving CTI block. However, the target power was not reached in up to $40 \%$ of the patients with an LTC. In that study, the maximum power was 32-35 Watts with an OITC, similar to the present study. However, the maximum power was limited to 50 watts with an LTC.

Another randomized study compared CTI ablation using an OITC versus an internally cooled catheter versus an $8 \mathrm{~mm}$ tip single sensor versus an $8 \mathrm{~mm}$ tip double sensor ablation catheter in 80 patients with atrial flutter. Maximum power was limited to 50 Watts with the open and internal irrigation catheters and 70 Watts with the large tip catheters. Patients were allowed to cross over to an alternate catheter after 12 minutes of ablation. The internally cooled catheter was found to be less effective than the other 3 catheters. There 
was no difference in efficacy between the LTC and OITC. However, tissue steam pops were observed with open and internally irrigated catheters. ${ }^{6}$

In a prior randomized study, ablation by targeting maximum voltage along the CTI was associated with a shorter duration of RF application than a conventional continuous ablation along the CTI. ${ }^{18}$ An 8-mm-tip catheter was used in both groups at a maximum power of 70 Watts.

In a prior nonrandomized study, both 8 - or $10-\mathrm{mm}$ tip catheters and a cooled-tip catheter were found to be associated with a higher acute efficacy and shorter procedure duration than a 4-mm tip catheter in achieving CTI block. ${ }^{19}$ There was no difference between the large-tip and cooled-tip catheters in acute procedural endpoints. However, unlike in the present study, clinical recurrence rate was higher after CTI ablation with a cooled-tip catheter.

\section{Limitations}

A limitation is that findings are applicable to the power settings used in this study. However, the settings used were based on the recommendations from the manufacturers and on existing literature. ${ }^{17}$ Although higher power settings may have improved efficiency with either catheter, the risk of adverse events may have been higher. ${ }^{6,20}$

A second limitation is that follow-up electrophysiologic testing to assess recovery of conduction across the CTI was not performed in this study. Therefore, it may not be possible to directly compare the prevalence of recovery of conduction between the 2 groups.

A third limitation is that cost comparison between the groups was not performed. The actual cost of catheters can be quite variable among different regions based on contractual agreements. However, due to the additional hardware needed for 3D-navigation and higher cost of an OITC than an LTC, CTI ablation using an OITC with 3D navigation is likely to be more costly than ablation using an LTC. The cost implications of continuing ablation with the same catheter versus switching to an alternate catheter if complete block could not be achieved within 20-30 minutes remains to be determined.

Another limitation of this study is that catheter systems using conventional RF energy that are available in the United States were compared in this study. It remains to be determined whether novel ablation systems and energy sources will have similar or superior efficacy than 10-mm tip catheters. ${ }^{21}$

\section{Clinical Implications}

Complete conduction block across the CTI can be achieved with $\leq 30$ minutes of ablation with either a largetip or an open irrigation tip catheter in $\sim 85 \%$ of patients with atrial flutter. However, complete block is achieved more quickly with an LTC than an OITC. Navigation of an OITC using a 3D electroanatomical mapping system does not appear to improve the efficiency or efficacy of CTI ablation compared to an LTC.

\section{References}

1. Perez FJ, Schubert CM, Parvez B, Pathak V, Ellenbogen KA, Wood MA: Long-term outcomes after catheter ablation of cavo-tricuspid isthmus dependent atrial flutter: A meta-analysis. Circ Arrhythm Electrophysiol 2009;2:393-401.
2. Cosio FG, Lopez-Gil M, Goicolea A, Arribas F, Barroso JL: Radiofrequency ablation of the inferior vena cava-tricuspid valve isthmus in common atrial flutter. Am J Cardiol 1993;71:705-709.

3. Feld GK: Radiofrequency catheter ablation of type 1 atrial flutter using a large-tip electrode catheter and high-power radiofrequency energy generator. Expert Rev Med Devices 2004;1:187-192.

4. Tada H, Oral H, Sticherling C, Chough SP, Baker RL, Wasmer K, Pelosi F Jr, Knight BP, Strickberger SA, Morady F: Double potentials along the ablation line as a guide to radiofrequency ablation of typical atrial flutter. J Am Coll Cardiol 2001;38:750-755.

5. Tsai CF, Tai CT, Yu WC, Chen YJ, Hsieh MH, Chiang CE, Ding YA, Chang MS, Chen SA: Is 8-mm more effective than 4-mm tip electrode catheter for ablation of typical atrial flutter? Circulation 1999;100:768771.

6. Scavee C, Jais P, Hsu LF, Sanders P, Hocini M, Weerasooriya R, Macle L, Raybaud F, Clementy J, Haissaguerre M: Prospective randomised comparison of irrigated-tip and large-tip catheter ablation of cavotricuspid isthmus-dependent atrial flutter. Eur Heart J 2004;25: 963-969.

7. Da Costa A, Faure E, Thevenin J, Messier M, Bernard S, Abdel K, Robin C, Romeyer C, Isaaz K: Effect of isthmus anatomy and ablation catheter on radiofrequency catheter ablation of the cavotricuspid isthmus. Circulation 2004;110:1030-1035.

8. Jais P, Shah DC, Haissaguerre M, Hocini M, Garrigue S, Le Metayer P, Clementy J: Prospective randomized comparison of irrigated-tip versus conventional-tip catheters for ablation of common flutter. Circulation 2000;101:772-776.

9. Kasai A, Anselme F, Teo WS, Cribier A, Saoudi N: Comparison of effectiveness of an 8-mm versus a 4-mm tip electrode catheter for radiofrequency ablation of typical atrial flutter. Am J Cardiol 2000;86:1029-1032, A1010.

10. Da Costa A, Jamon Y, Romeyer-Bouchard C, Thevenin J, Messier $\mathrm{M}$, Isaaz K: Catheter selection for ablation of the cavotricuspid isthmus for treatment of typical atrial flutter. J Interv Card Electrophysiol 2006;17:93-101.

11. Da Costa A, Romeyer-Bouchard C, Jamon Y, Bisch L, Isaaz K: Radiofrequency catheter selection based on cavotricuspid angiography compared with a control group with an externally cooled-tip catheter: A randomized pilot study. J Cardiovasc Electrophysiol 2009;20:492498.

12. Da Costa A, Romeyer-Bouchard C, Dauphinot V, Lipp D, Abdellaoui L, Messier M, Thevenin J, Barthelemy JC, Isaaz K: Cavotricuspid isthmus angiography predicts atrial flutter ablation efficacy in 281 patients randomized between $8 \mathrm{~mm}$ - and externally irrigated-tip catheter. Eur Heart J 2006;27:1833-1840.

13. Cabrera JA, Sanchez-Quintana D, Ho SY, Medina A, Anderson RH: The architecture of the atrial musculature between the orifice of the inferior caval vein and the tricuspid valve: The anatomy of the isthmus. J Cardiovasc Electrophysiol 1998;9:1186-1195.

14. Hindricks G, Willems S, Kautzner J, De Chillou C, Wiedemann M, Schepel S, Piorkowski C, Risius T, Kottkamp H: Effect of electroanatomically guided versus conventional catheter ablation of typical atrial flutter on the fluoroscopy time and resource use: A prospective randomized multicenter study. J Cardiovasc Electrophysiol 2009;20:734-740

15. Hall B, Veerareddy S, Cheung P, Good E, Lemola K, Han J, Kamala T, Chugh A, Pelosi F Jr, Morady F, Oral H: Randomized comparison of anatomical versus voltage guided ablation of the cavotricuspid isthmus for atrial flutter. Heart Rhythm 2004;1:43-48.

16. Nakagawa H, Yamanashi WS, Pitha JV, Arruda M, Wang X, Ohtomo K, Beckman KJ, McClelland JH, Lazzara R, Jackman WM: Comparison of in vivo tissue temperature profile and lesion geometry for radiofrequency ablation with a saline-irrigated electrode versus temperature control in a canine thigh muscle preparation. Circulation 1995;91:22642273.

17. Sacher F, O'Neill MD, Jais P, Huffer LL, Laborderie J, Derval N, Deplagne A, Takahashi Y, Jonnson A, Hocini M, Clementy J, Haissaguerre M: Prospective randomized comparison of 8-mm gold-tip, externally irrigated-tip and $8-\mathrm{mm}$ platinum-iridium tip catheters for cavotricuspid isthmus ablation. J Cardiovasc Electrophysiol 2007;18:709-713

18. Gula LJ, Redfearn DP, Veenhuyzen GD, Krahn AD, Yee R, Klein GJ, Skanes AC: Reduction in atrial flutter ablation time by targeting maximum voltage: Results of a prospective randomized clinical trial. J Cardiovasc Electrophysiol 2009;20:1108-1112.

19. Marrouche NF, Schweikert R, Saliba W, Pavia SV, Martin DO, Dresing T, Cole C, Balaban K, Saad E, Perez-Lugones A, Bash D, Tchou P, 
Natale A: Use of different catheter ablation technologies for treatment of typical atrial flutter: Acute results and long-term follow-up. Pacing Clin Electrophysiol 2003;26:743-746.

20. Hillock RJ, Melton IC, Crozier IG: Radiofrequency ablation for common atrial flutter using an 8-mm tip catheter and up to $150 \mathrm{~W}$. Europace $2005 ; 7: 409-412$.
21. Erdogan A, Guettler N, Doerr O, Franzen W, Soydan N, Bilgin M, Vogelsang P, Parahuleva M, Tillmanns H, Stracke S, Guenduez D, Neuhof C: Randomized comparison of multipolar, duty-cycled, bipolar-unipolar radiofrequency versus conventional catheter ablation for treatment of common atrial flutter. J Cardiovasc Electrophysiol 2010;21:1109-1113. 